\title{
Series: Cardiovascular outcome trials for diabetes drugs Alogliptin and EXAMINE
}

\author{
MILES FISHER
}

\begin{abstract}
EXAMINE was an FDA mandated cardiovascular outcome trial with alogliptin. In contrast to other cardiovascular outcome trials with DPP-4 inhibitors, it was performed in subjects with a recent acute coronary syndrome. EXAMINE compared alogliptin and placebo in 5,380 subjects with type 2 diabetes and demonstrated non-inferiority for major cardiovascular events (cardiovascular death, myocardial infarction, stroke) but not superiority. Data on hospitalisation for heart failure were not included in the principal publication. A subsequent publication showed no overall increase in hospitalisation for heart failure with alogliptin, but when subjects with and without baseline heart failure were separated there was a significant increase in the group without heart failure at baseline. No clear clinical benefit has been established for alogliptin, and there are alternatives such as sitagliptin and linagliptin that are not associated with an increase in hospitalisation for heart failure.
\end{abstract}

Br J Diabetes 2019;19:133-135

Key words: diabetes, cardiovascular outcome trial, alogliptin

\section{Introduction}

Licensing requirements for new anti-diabetes drugs changed in the USA and Europe in 2008 and 2012.1,2 The phase III development programme was required to include participants that were more representative of the wider diabetes population, cardiovascular events occurring in the phase III development programme were to be blindly adjudicated to provide information on cardiovascular safety, and a dedicated randomised controlled cardiovascular outcome trial (CVOT) was usually required either before or after licensing. This is the second article in a series which describes and summarises the results of each of these CVOTs in the chronological order in which they were published, describing the primary endpoint and important secondary outcomes from the principal publication, but also directs attention to important subsequent publications of data from subgroups and post hoc analyses.

Address for correspondence: Miles Fisher Department of Diabetes, Endocrinology \& Clinical Pharmacology, Glasgow Royal Infirmary, 84 Castle St, Glasgow G4 OSF, UK

E-mail: miles.fisher@ggc.scot.nhs.uk

https://doi.org/10.15277/bjd.2019.230

\section{Background}

The DPP-4 inhibitor alogliptin was licensed in 2013 by the FDA for use in the USA and by the EMA for use in Europe. A systematic assessment of cardiovascular outcomes in the phase II and phase III trials in the development programme was published in early 2013. ${ }^{3}$ Major adverse cardiovascular events (MACE; cardiovascular death, non-fatal myocardial infarction, non-fatal stoke) were adjudicated by an expert endpoint committee blinded to treatment allocation. A total of 13 MACE events were adjudicated in 4,168 patients receiving alogliptin and 10 MACE events were identified in 1,860 patients randomised to comparator therapies, so the incidence rates of MACE were not significantly different between patients treated with alogliptin and comparator therapies. In addition, 10 non-MACE cardiovascular events (angina, arrhythmias, heart failure) occurred with alogliptin and three non-MACE cardiovascular events occurred in patients randomised to the comparator therapies (NS). The number of heart failure events as a single outcome was not described.

\section{EXAMINE}

A paper describing the design and rationale of EXAMINE was published in 2011. ${ }^{4}$ The primary endpoint was detailed as MACE. Hospitalisation for heart failure was not included as a single endpoint, but it was included as a component of a so-called exploratory MACE composite of all-cause mortality, non-fatal myocardial infarction, non-fatal stroke, urgent revascularisation for unstable angina, and hospitalisation for heart failure. ${ }^{4}$ The principal EXAMINE results were presented in 2013 at the meeting of the European Society of Cardiology (ESC) and published simultaneously in the New England Journal of Medicine (NEJM). ${ }^{5}$ The design of the study and key baseline characteristics are described in Box 1. In EXAMINE there was no significant difference in MACE, so non-inferiority was established but not superiority (Figure 1, Box 2). No data on hospitalisation for heart failure were presented at the ESC or published in the NEJM. It is surprising that the editors of the NEJM did not request heart failure results as data on hospitalisation for heart failure were included in the publication of SAVOR-TIMI with saxagliptin in the same edition of the NEJM, and have demonstrated a significant increase in hospitalisation for heart failure in the saxagliptin group. ${ }^{6}$

Data on hospitalisation for heart failure were finally published in the Lancet more than one year later. ${ }^{7}$ The analyses presented included the data for hospital admission for heart failure as part of the exploratory MACE composite, and a composite of cardiovascular death and hospitalisation for heart failure. The criteria to 


\section{Box 1 Key features of EXAMINE 4,5}

- EXAMINE compared alogliptin versus placebo for a median of 18 months in 5,380 subjects

- Median age of subjects was 61 years with a median duration of diabetes of 7 years

- Mean baseline $\mathrm{HbA}_{1 \mathrm{c}}$ was $8.0 \%$ (64 $\left.\mathrm{mmol} / \mathrm{mol}\right)$

- $100 \%$ of subjects had an acute coronary syndrome within 15-90 days before randomisation, $88 \%$ myocardial infarction including the index event of acute coronary syndrome, $63 \%$ prior percutaneous coronary intervention including the index case and $28 \%$ investigator reported heart failure at baseline

- $66 \%$ of subjects were on metformin, $46 \%$ sulfonylureas, $2 \%$ thiazolidinediones, $30 \%$ insulin

Figure 1. Eighteen-month estimated event rates (in \%) comparing alogliptin and placebo for major adverse cardiovascular events (MACE), total mortality, cardiovascular mortality, non-fatal myocardial infarction, non-fatal stroke and hospitalisation for heart failure $(\mathrm{HFH})$

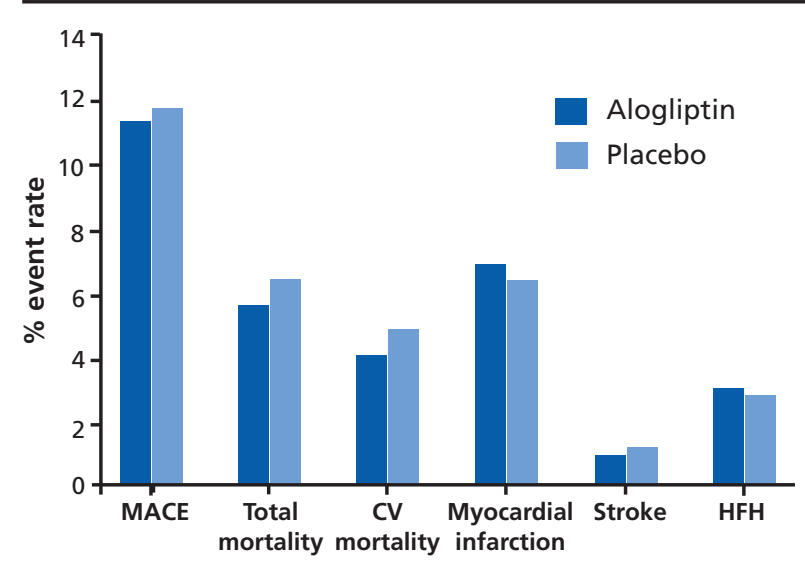

define hospital admission for heart failure were an inpatient admission or an emergency department visit of more than 12 hours with clinical manifestations of heart failure, including new or worsening dyspnoea, orthopnoea, paroxysmal nocturnal dyspnoea, peripheral oedema, bibasilar rales on pulmonary examination, jugular venous distention, new third heart sound, radiographic evidence of heart failure and parenteral diuretic, inotropic or vasodilator therapy, ultrafiltration or dialysis, or mechanical or surgical intervention (including heart transplant).

No differences were seen in the exploratory extended MACE endpoint in heart failure as a single endpoint or in the composite of cardiovascular death and hospitalisation for heart failure. However, post hoc analyses showed a significant increase in hospitalisation for heart failure subjects with no history of heart failure at baseline (2.2\% vs. $1.3 \%$, hazard ratio 1.76 (95\% Cl 1.07 to $1.42, \mathrm{p}=0.026)$. This result is counterintuitive and does not make biological sense as it would be expected that heart failure might be increased in subjects who had a history of baseline heart failure, as was seen in SAVOR-TIMI. ${ }^{6}$

\section{Box 2 Results of the EXAMINE trial}

\section{Principal result}

- No reduction in $\mathrm{MACE}^{5}$

\section{Other results from EXAMINE}

- Reported events of hypoglycaemia and serious hypoglycaemia were associated with MACE

- Addition of alogliptin to dual therapy with metformin plus sulfonylurea significantly reduced $\mathrm{HbA}_{1 \mathrm{c}}$ and was well tolerated ${ }^{14}$

- Baseline adiponectin concentration was independently associated with increased risk of death from CV causes, all-cause mortality and hospitalisation for heart failure ${ }^{15}$

- Levels of high-sensitivity C-reactive protein were associated with recurrent cardiovascular events and this appeared to be independent of the achieved LDL cholesterol concentration ${ }^{16}$

- The addition of cystatin C or biomarkers of tubular injury did not improve the prediction of eGFR decline beyond common clinical factors and routine laboratory data ${ }^{17}$

- A strong relationship was observed between baseline and 6-month NT-proBNP concentrations and incident major cardiovascular events, particularly hospitalisation for heart failure ${ }^{18}$

- Serial measurement of high-sensitivity troponin I revealed a proportion of patients without clinically recognised events had dynamic or persistently raised values and were at high risk of recurrent events ${ }^{19}$

- In EXAMINE, average clinician-measured blood pressure less than $130 / 80 \mathrm{mmHg}$ was associated with worsened cardiovascular outcomes 20

\section{Other results from EXAMINE}

Further publications from EXAMINE are detailed in Box 2. The most important of these was an analysis of the relationship of glycated haemoglobin and reported hypoglycaemia to cardiovascular outcomes. ${ }^{8}$ No change in rates of MACE was observed according to baseline $\mathrm{HbA}_{1} \mathrm{c}$. Patients in the alogliptin arm achieved lower $\mathrm{HbA}_{1 \mathrm{c}}$ levels than in the control group with no difference in the rates of hypoglycaemia. Combining the alogliptin and placebo groups, patients who experienced serious hypoglycaemia had a higher MACE rate than those who did not, similar to observations from the ADVANCE trial. ${ }^{9}$

\section{Discussion}

EXAMINE was the second published FDA mandated cardiovascular outcome trial with a new diabetes drug. It showed that alogliptin had no effect on atherosclerotic endpoints. Like the increase in hospitalisation for heart failure that was seen in the SAVOR-TIMI trial with saxagliptin, the possible increase in hospitalisation for heart failure in a subgroup in EXAMINE was unexpected and the mechanisms remain unclear. No increase in hospitalisation for heart failure was seen in the subsequent TECOS trial with sitagliptin, ${ }^{10}$ or the CARMELINA and CAROLINA trials with linagliptin. ${ }^{11,12}$ For patients with existing heart failure, or those who are at a high risk of developing heart failure including following an acute coronary syndrome, other alternatives are available, including SGLT2 inhibitors, which significantly reduce heart failure outcomes in people with diabetes. ${ }^{13}$ 


\section{Key messages}

- EXAMINE was the second published cardiovascular outcome trial of a diabetes drug, comparing alogliptin and placebo

- In EXAMINE, alogliptin had no effect on atherosclerotic events of cardiovascular death, myocardial infarction or stroke

- An increase in hospitalisation for heart failure was observed in a subgroup who did not have heart failure at baseline, and the mechanism of this increase remains uncertain

- For patients with existing heart failure or who are at high risk of developing failure, SGLT2 inhibitors are a better alternative

Conflict of interest: The author has received payment for advisory boards and/or lectures from Astra Zeneca, Boehringer Ingelheim, Eli Lilly, Janssen, MSD, NAPP, Novartis, Novo Nordisk, Sanofi, Takeda.

\section{Funding: None.}

\section{References}

1. Food and Drug Administration. Guidance for industry. Diabetes mellitus - evaluating cardiovascular risk in new antidiabetic therapies to treat type 2 diabetes. Silver Spring, MD: US Department of Health and Human Services, Food and Drug Administration, Center for Drug Evaluation and Research (CDER), 2008. Available from: https://www.fda.gov/regulatoryinformation/search-fda-guidance-documents/diabetes-mellitus-evaluating-cardiovascular-risk-new-antidiabetic-therapies-treat-type-2-diabetes (accessed 8th Nov 2019).

2. European Medicines Agency. Guideline on clinical investigation of medicinal products in the treatment or prevention of diabetes mellitus. London: EMA, 2012. Available from: http://www.ema.europa.eu/docs/en _GB/document_library/Scientific_guideline/2012/06/WC500129256.pdf (accessed 9 Feb 2019).

3. White WB, Pratley R, Fleck P, et al. Cardiovascular safety of the dipeptidyl peptidase-4 inhibitor alogliptin in type 2 diabetes mellitus. Diabetes Obes Metab 2013;15:668-73. https://doi.org/10.1111/dom.12093

4. White WB, Bakris GL, Bergenstal R, et al. EXamination of cArdiovascular outcoMes with AlogliptIN versus Standard of CarE in patients with type 2 diabetes mellitus and acute coronary syndrome (EXAMINE): a cardiovascular safety study of the dipeptidyl peptidase 4 inhibitor alogliptin in patients with type 2 diabetes with acute coronary syndrome. Am Heart J 2011;162:620-6. https://doi.org/10.1016/j.ahj.2011.08.004

5. White WB, Cannon CP, Heller SR, et al, for the EXAMINE Investigators. Alogliptin after acute coronary syndrome in patients with type 2 diabetes. N Engl J Med 2013;369:1327-35. https://doi.org/10.1056/NEJMoa1305889

6. Scirica BM, Bhatt DL, Braunwald E, et al, for the SAVOR-TIMI 53 Steering Committee and Investigators. Saxagliptin and cardiovascular outcomes in patients with type 2 diabetes mellitus. N Engl J Med 2013;369:1317-
26. https://doi.org/10.1056/NEJMoa1307684

7. Zannad F, Cannon CP, Cushman WC, et al, for the EXAMINE Investigators. Heart failure and mortality outcomes in patients with type 2 diabetes taking alogliptin versus placebo in EXAMINE: a multicentre, randomised, double-blind trial. Lancet 2015;385:2067-76. https://doi.org/10.1016/S0140-6736(14)62225-X

8. Heller SR, Bergenstal RM, White WB, et al, for the EXAMINE Investigators. Relationship of glycated haemoglobin and reported hypoglycaemia to cardiovascular outcomes in patients with type 2 diabetes and recent acute coronary syndrome events: the EXAMINE trial. Diabetes Obes Metab 2017;19:664-71. https://doi.org/10.1111/dom.12871

9. Zoungas Z, Patel A, Chalmers J, et al, for the ADVANCE Collaborative Group. Severe hypoglycaemia and risks of vascular events and death. $N$ Engl J Med 2010;363:1410-18. https://doi.org/10.1056/ NEJMoa1003795

10. Green JB, Bethel A, Armstrong PW, et al, for the TECOS Study Group. Effect of sitagliptin on cardiovascular outcomes in type 2 diabetes. $N$ Engl J Med 2015;373:232-42. https://doi.org/10.1056/NEJMoa1501352

11. Rosenstock J, Perkovic $V$, Johansen OE, et al, for the CARMELINA Investigators. Effects of linagliptin vs placebo on major cardiovascular events in adults with type 2 diabetes and high cardiovascular and high renal risk. The CARMELINA randomized clinical trial. JAMA 2019;321:69-79. https://doi.org/10.1001/jama.2018.18269

12. Rosenstock J, Kahn SE, Johansen OE, et al, for the CAROLINA Investigators. Effect of linagliptin vs glimepiride on major adverse cardiovascular outcomes in patients with type 2 diabetes. The CAROLINA randomized clinical trial. JAMA 2019 Sep 19 [Epub ahead of print]. https://doi.org/10.1001/jama.2019.13772

13. Zelniker TA, Wiviott SD, Raz I, et al. SGLT2 inhibitors for primary and secondary prevention of cardiovascular and renal outcomes in type 2 diabetes: a systematic review and meta-analysis of cardiovascular outcome trials. Lancet 2019;393:31-9. https://doi.org/10.1016/S01406736(18)32590-X

14. White WB, Heller SR, Cannon $C P$, et al, for the EXAMINE Investigators. Alogliptin in patients with type 2 diabetes receiving metformin and sulfonylurea therapies in the EXAMINE trial. Am J Med 2018;131:81319.e5. https://doi.org/10.1016/j.amjmed.2018.02.023

15. Bergmark BA, Cannon CP, White WB, et al. Baseline adiponectin concentration and clinical outcomes among patients with diabetes and recent acute coronary syndrome in the EXAMINE trial. Diabetes Obes Metab 2017;19:962-9. https://doi.org/10.1111/dom.12905

16. Whang $\mathrm{Y}-\mathrm{C}$, Morrow DA, Cannon $\mathrm{CP}$, et al. High-sensitivity $\mathrm{C}$-reactive protein, low-density lipoprotein cholesterol and cardiovascular outcomes in patients with type 2 diabetes in the EXAMINE (Examination of Cardiovascular Outcomes with Alogliptin versus Standard of Care) trial. Diabetes Obes Metab 2018;20:654-9. https://doi.org/10.1111/ dom.13136

17. Garlo KG, White WB, Bakris GL, et al. Kidney biomarkers and decline in eGFR in patients with type 2 diabetes. Clin J Am Soc Nephrol 2018;13:398-405. https://doi.org/10.2215/CJN.05280517

18. Jarolim $P$, White WB, Cannon CP, et al. Serial measurement of natriuretic peptides and cardiovascular outcomes in patients with type 2 diabetes in the EXAMINE trial. Diabetes Care 2018;41:1510-15. https://doi.org/ $10.2337 / \mathrm{dc} 18-0109$

19. Cavender MA, White WB, Jarolim $P$, et al. Serial measurement of highsensitivity troponin I and cardiovascular outcomes in patients with type 2 diabetes mellitus in the EXAMINE trial (Examination of Cardiovascular Outcomes with Alogliptin versus Standard of Care). Circulation 2017; 135:1911-21. https://doi.org/10.1161/CIRCULATIONAHA.116.024632

20. White WB, Jalil F, Cushman WC, et al. Average clinician-measured blood pressures and cardiovascular outcomes in patients with type 2 diabetes mellitus and ischemic heart disease in the EXAMINE Trial. J Am Heart Assoc 2018;7:e009114. https://doi.org/10.1161/JAHA.118.009114 